\title{
THE EFFICIENCY OF HAPLOID METHOD OF PLANT BREEDING
}

\author{
MASATOSHI NEI \\ Division of Genetics. National Institute of Radiological Sciences, Chiba, Japan
}

\section{INTRODUCTION}

Received 20.vii.62

IN the conventional method of plant breeding a considerable number of generations are needed for obtaining pure lines from a heterozygous population. If, however, the chromosomes of haploids, gametophytic or sporophytic, are doubled, completely homozygous lines can be obtained in one or two generations. This is one of the main reasons why so many geneticists have devoted themselves to the artificial production of haploids, although none of them has succeeded in developing a method applicable to every sort of plants. In such crops as corn, potato and einkorn wheat, however, the frequency of haploid occurrence can be increased to a great extent by certain specific techniques (Katayama, 1934; Kihara, 1940; Chase, 1952; Peloquin and Hougas, 1959). A promisingly high frequency of haploids (3.2 per cent.) was recently obtained by Coe (1959) in corn with use of a selected pollinator. According to Dr W. L. Brown (oral communication) Chase is now using the haploid method for obtaining homozygous lines in corn breeding. The purpose of this paper is to examine the efficiency of this method in comparison with the conventional diploid method.

\section{SELF-FERTILISING PLANTS}

If the $\mathrm{F}_{1}$ plants from a cross are treated with some technique for the induction of haploid parthenogenesis, completely homozygous lines can be obtained in the $F_{3}$ generation as given in fig. I (orthodox

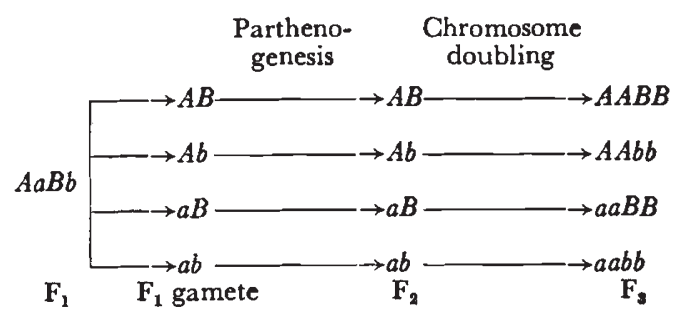

Fic. 1.-The orthodox haploid method of breeding in self-fertilising plants.

haploid method). On the other hand, if pseudo-diploid parthenogenesis ${ }^{*}$ ) is induced, the homozygous lines are obtained in the $\mathrm{F}_{2}$

* Pseudo-diploid parthenogenesis refers to the parthenogenetic development of $2 n$ sporophytes from a chromosome-doubled in egg cell not from a $2 n$ egg cell. 
generation as was reported by East (1930), Terao (1934) and others.

Now let us consider the efficiency of selection in the $F_{2}$ generation. In haploid method (pseudo-diploid parthenogenesis in this case) there occur two kinds of genotypes for a pair of alleles, $A$ and $a$, with the frequencies of $\frac{1}{2} A A$ and $\frac{1}{2} a a$, while in diploid method three genotypes occur with the frequencies of $\frac{1}{4} A A, \frac{1}{2} A a$ and $\frac{1}{4} a a$. Thus, if $A A$ is a desirable genotype, the probability of obtaining this genotype is higher in haploid method than in diploid method, as was already pointed out by Terao (1934) and Chase (1952). In the case of $n$ loci segregating independently, the probability of obtaining the desirable genotype is $\left(\frac{1}{2}\right)^{n}$ in haploid method and $\left(\frac{1}{4}\right)^{n}$ in diploid method. At present, however, the haploid method has a great drawback in another respect. That is, the frequencies of parthenogenetic development of egg cells $\left(p_{1}\right)$ and chromosome doubling of haploid gametophytes or sporophytes $\left(p_{2}\right)$ are generally very low. Therefore, taking into account this factor the efficiency of haploid method over diploid method may be defined as

$$
\mathrm{E}=\frac{p_{1} p_{2}\left(\frac{1}{2}\right)^{n}}{\left(\frac{1}{4}\right)^{n}}=p_{1} p_{2} 2^{n}
$$

It is clear from this formula that for haploid method to be more efficient than diploid method $\mathrm{E}$ must be larger than $\mathrm{I}$ and therefore

$$
p_{1} p_{2}>\left(\frac{1}{2}\right)^{n}
$$

Thus, if a large number of genes are concerned, the haploid method seems to be superior to the diploid method, even if $p_{1} p_{2}$ is small. The value of $\overline{p_{1} p_{2}}=\left(\frac{1}{2}\right)^{n}$ will be a criterion of whether the haploid method is more efficient than the diploid method or not.

In the above formulation, however, we have neglected the effect of linkage. The mathematical formulation to take into account the effect of linkage is very difficult, because we have little or no information about the distribution of segregating genes on the chromosomes in actual plant breeding. The only thing we know is that there are probably a large number of genes concerned with yield or many other quantitative characters under selection, perhaps several-fold the chromosome number. The reduction in linkage disequilibrium under selfing is very small compared with that under random mating, the largest reduction being obtained from the $F_{1}$ to $F_{2}$ generation. Thus, if we neglect the recombination in the $F_{2}$ and its subsequent generations, the formula equivalent to (2) becomes

$$
p_{1} p_{2}>\left(\frac{1}{2}\right)^{n^{\prime}+n^{*}}
$$

where $n^{\prime}$ is the chromosome number in haploid phase and $n^{\prime \prime}$ the total number of chiasmata on the whole chromosomes.

Formula (3) seems to have wide applicability with a relatively high approximation, if the number of chiasmata can be determined from a cytological observation. If the number of chiasmata is not 
known, a further approximation may be made by simply taking $n$ as the chromosome number in formula (2). In this case $\overline{p_{1} p_{2}}$ is evaluated higher than necessary, and therefore it seems a safe criterion for the evaluation of haploid method. Taking this for granted, we have $\overline{p_{1} p_{2}}=0.78$ per cent. for barley $(n=7), 0.097$ per cent. for

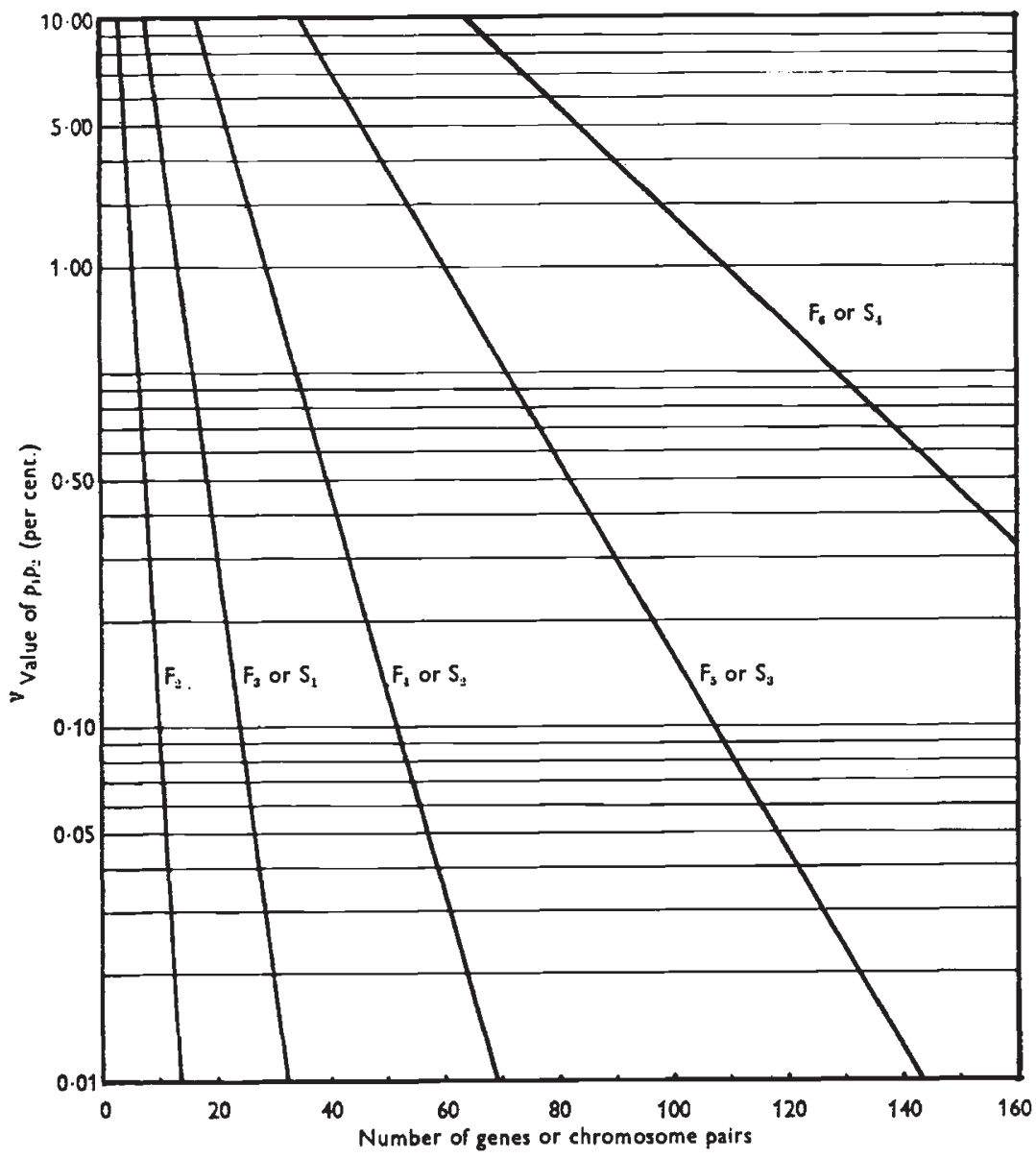

FIG. 2.-Values of $p_{1} p_{8}$ necessary for the haploid method of plant breeding to be as efficient as the conventional method. The frequency of the favourable allele at each locus is assumed to be one-half in all cases.

corn $(n=10), 0.0048$ per cent. for bread wheat $(n=2 \mathrm{I})$. The general relation between $n$ and $\overline{p_{1} p_{2}}$ is given in fig. $2\left(\mathrm{~F}_{2}\right)$.

Terao reported that in Brassica napus $(n=19)$ the frequency of parthenogenetic homozygous diploids amounts to 7.62 per cent. per flower pollinated when crossed with Eruca sativa. Since one capsule of $B$. napus contains about 25 seeds on the average, $p_{1} p_{2}$ is approximately 0.305 per cent., which far exceeds the critical value of $\left(\frac{1}{2}\right)^{19}=0.0019$ 
per cent. Thus, in this situation the haploid method is likely to be more efficient than diploid method.

Next consider the case of fig. I in which the homozygous lines are obtained in the $F_{3}$ generation. In this case the value of $p_{1} p_{2}$ required for haploid method to be more efficient than diploid method is

$$
p_{1} p_{2}>\left(\frac{3}{4}\right)^{n}
$$

where $n$ is either the number of loci or chromosome pairs. It is seen from this formula that the haploid method is less efficient in this case than in the above, but if the number of loci or chromosome pairs concerned is large, the haploid method is still not necessarily inferior to the diploid method, even if $p_{1} p_{2}$ is small. The relation between $n$ and $\overline{p_{1} p_{2}}$ is given in fig. $2\left(\mathrm{~F}_{3}\right)$.

In some situations the treatment for inducing parthenogenesis may be made in the $F_{2}$ or its subsequent generations, homozygous diploids being secured two generations after the treatment. The general formula for the critical value of $p_{1} p_{2}$ is

$$
\overline{p_{1} p_{2}}=\left(\frac{2^{g-1}-\mathrm{I}}{2^{g-1}}\right)^{n}
$$

where $g$ refers to the $\mathrm{F}_{g}$ generation. Thus, as the generation proceeds, the advantage of the haploid method rapidly decreases. This is easily seen from fig. 2.

\section{CROSS-FERTILISING PLANTS}

In a random mating population linkage disequilibrium may be assumed to be o irrespective of the distribution of genes on the chromosomes, unless there are certain types of epistasis. Thus, assuming no epistasis, we have the following formula for $p_{1} p_{2}$.

$$
p_{1} p_{2}>\prod_{k=1}^{n} \frac{\mathrm{I}}{2^{g}}\left(2^{g}-\mathrm{I}+q_{k}\right)
$$

where $g$ refers to the $\mathrm{S}_{\boldsymbol{g}}$ generation, $n$ is the number of genes and $q_{k}$ the frequency of the favourable allele at locus $k$, no matter how many alleles are involved. Formula (6) reduces to

$$
p_{1} p_{2}>\prod_{k=1}^{n} \frac{\mathrm{I}}{2}\left(\mathrm{I}+q_{k}\right)
$$

for the case of pseudo-diploid parthenogenesis $\left(S_{1}\right)$, and to

$$
p_{1} p_{2}>\prod_{k=1}^{n} \frac{\mathrm{I}}{4}\left(3+q_{k}\right)
$$

or the orthodox haploid method $\left(\mathrm{S}_{2}\right)$.

As seen from the above formulæ, the haploid method is less efficient in this case than in the case of self-fertilising plants, if the 
$q$ 's are all one-half. This is because $\mathrm{S}_{0}$ corresponds to $\mathrm{F}_{2}$ with respe ct to the inbreeding coefficient. This disadvantage, however, is offset by the linkage equilibrium which may be expected in random mating populations, provided that a large number of genes are concerned. For example, in the orthodox haploid method, if $n=50$, the critical value of $p_{1} p_{2}$ is 0.102 per cent., as will be seen from fig. 2, and if $n=\mathrm{I} 00$, the critical value becomes $0.000 \mathrm{I} 6$ per cent. As mentioned before, haploids can be secured in corn with the frequency of 3.2 per cent. $\left(p_{1}\right)$ and ro per cent. $\left(p_{2}\right)$ of them produce spontaneous diploid sectors (Chase). Thus, the haploid method is not necessarily disadvantageous in corn breeding.

The efficiency of the haploid method is obviously high when the number of genes concerned is large and the frequencies of favourable alleles are small, if $p_{1} p_{2}$ is constant. This is because this method is a kind of gamete selection.

\section{DISCUSSION}

In the above comparison of the haploid and diploid methods, we have neglected the effect of selection during the period of selfing, which is possible only in the diploid method. This effect of selection seems to be high for highly heritable characters such as plant height and flowering time and consequently makes the diploid method more advantageous than it appears in the above formulation. However, when we turn to yield selection in early generations appears very ineffective at least for self-fertilising plants (Nei and Matzinger, unpublished). As for cross-fertilising plants Thompson made in corn the comparison of combining abilities of homozygous diploid lines obtained by the haploid method and inbred lines which were subjected to top cross selection in early generations, but he could not find any substantial difference between the two groups. Thus, the present formulation does not seem to lead to any serious error.

The use of the haploid method should be of particular value in forest tree breeding. Forest trees usually take at least several years to flower and consequently a long time for obtaining inbred lines. This shortcoming has made breeders pessimistic about the utilisation of heterosis in forest trees. The best method to overcome the shortcoming is to use the haploid method.

\section{SUMMARY}

The efficiency of the haploid method of plant breeding is examined in comparison with the conventional diploid method. The frequencies of haploid occurrence and its diploidisation that are necessary for the haploid method to be more efficient than the diploid method are calculated. It is concluded that the haploid method is advantageous when the number of genes concerned is large and the frequencies of favourable alleles in populations are small, even if the frequency of 
haploid occurrence is small. It is also noted that the haploid method offers a time-saving approach to the utilisation of heterosis in forest tree breeding.

\section{REFERENCES}

CoE, E. H. 1959. A line of maize with high haploid frequency. Amer. Nat., 93, $3^{8} \mathrm{r}-3^{82}$.

Chase, s. s. 1952. Monoploids in maize. Heterosis (ed. by J. W. Gowen), pp. 389399.

EAST, E. M. I930. The production of homozygotes through induced parthenogenesis. Science, 72, 148-149.

KATAYAMA, Y. I934. Haploid formation by X-rays in Triticum monococcum. Cytologia, $5,235-237$.

KIHARA, H. I940. Formation of haploids by means of delayed pollination in Triticum monococcum. Bot. Mag., 54, 178-185.

NEI, M., AND MATZINGER, D. F. Comparative evaluation of breeding procedures in self-fertilising plants. North Carolina State Coll. Bull. In press.

PELOQUIN, s. J., AND hoUGAs, R. W. I959. Decapitation and genetic markers as related to haploidy in Solanum tuberosum. Europ. potato J., 2, 176-183.

TERAO, H. 1934. Induction of parthenogenesis by means of interspecific crosses and its significance for plant breeding. Agr. and Hort., 9, I-10. Japanese.

Thompson, D. L. 1954. Combining ability of homozygous diploids of corn relative to lines derived by inbreeding. Agron. F., $46,133-136$. 